\title{
Infant discrimination of humanoid robots
}

\author{
Goh Matsuda ${ }^{1,2}$, Hiroshi Ishiguro ${ }^{3,4}$ and Kazuo Hiraki2,5* \\ ${ }^{1}$ Department of Medical Education and General Medicine, Kyoto Prefectural University of Medicine, Kyoto, Japan, \\ ${ }^{2}$ Department of General Systems Studies, Graduate School of Arts and Sciences, The University of Tokyo, Tokyo, Japan, \\ ${ }^{3}$ Department of Systems Innovation, Graduate School of Engineering Science, Osaka University, Osaka, Japan, ${ }^{4}$ Hiroshi \\ Ishiguro Laboratories, Advanced Telecommunications Research Institute International, Kyoto, Japan, ${ }^{5}$ CREST - Japan \\ Science and Technology Agency, Tokyo, Japan
}

Recently, extremely humanlike robots called "androids" have been developed, some of which are already being used in the field of entertainment. In the context of psychological studies, androids are expected to be used in the future as fully controllable human stimuli to investigate human nature. In this study, we used an android to examine infant discrimination ability between human beings and non-human agents. Participants ( $N=42$ infants) were assigned to three groups based on their age, i.e., 6- to 8month-olds, 9- to 11-month-olds, and 12- to 14-month-olds, and took part in a preferential looking paradigm. Of three types of agents involved in the paradigm-a human, an android modeled on the human, and a mechanical-looking robot made from the android-two at a time were presented side-by-side as they performed a grasping action. Infants' looking behavior was measured using an eye tracking system, and the amount of time spent focusing on each of three areas of interest (face, goal, and body) was analyzed. Results showed that all age groups predominantly looked at the robot and at the face area, and that infants aged over 9 months watched the goal area for longer than the body area. There was no difference in looking times and areas focused on between the human and the android. These findings suggest that 6- to 14-montholds are unable to discriminate between the human and the android, although they can distinguish the mechanical robot from the human.

ent General Syste

Studies, Graduate School of Arts and Sciences, The University of Tokyo, 3-8-1 Komaba, Meguro-ku, Tokyo 153-8902, Japan khiraki@idea.c.u-tokyo.ac.jp

Specialty section: This article was submitted to Human-Media Interaction, a section of the journal Frontiers in Psychology

Received: 15 July 2015 Accepted: 02 September 2015 Published: 22 September 2015

Citation:

Matsuda G, Ishiguro H and Hiraki K (2015) Infant discrimination of humanoid robots.

Front. Psychol. 6:1397. doi: 10.3389/fpsyg.2015.01397

\section{Keywords: infant, humanoid robot, android, preferential looking paradigm, eye tracking, uncanny valley}

\section{Introduction}

Over the last decade, various types of humanoid robots have emerged beyond the hypothetical realm of science fiction and into real life. More recently, robots with an extremely humanlike appearance, called "androids," were developed (Ishiguro, 2006), primarily for interaction with humans. Because the best communicative partner of human beings is undoubtedly other humans, the development of a more humanlike appearance and motion for robots is considered a shortcut to developing robots that will have natural interactions with humans. Thus, investigating how currently available robots are perceived by humans will provide valuable information for this purpose.

The famous "uncanny valley" hypothesis is related to the impression conveyed by robots and their human likeness (Mori, 1970, 2012), and states that extremely humanlike artifacts often elicit negative affect, e.g., a feeling of eeriness, whereas modestly humanlike artifacts evoke familiarity. It was originally a theoretical hypothesis and remains controversial (Burleigh et al., 2013); some 
subsequent studies have, however, found empirical evidence supporting the existence of a similar phenomenon in both humans (Seyama and Nagayama, 2007) and other primates (Steckenfinger and Ghazanfar, 2009). In other words, the uncanny valley hypothesis suggests that humans have a sophisticated ability to discriminate between human and nonhuman beings. In fact, it has been reported that $80 \%$ of adult participants recognized that an android with a highly humanlike appearance was not a real human within 1 s (Noma et al., 2006), and that brain activity when viewing a human vs. an android is significantly different, especially in the anterior intraparietal sulcus, which is involved in action perception (Saygin et al., 2011). Currently available androids, therefore, do not seem to have achieved a sufficiently humanlike appearance in the view of human adults.

On the other hand, little is known about infant perception of extremely humanlike artifacts, such as androids. Newborns show primary discrimination abilities in relation to human properties, such as faces, voices, and movements (Goren et al., 1975; DeCasper and Fifer, 1980; Moon et al., 1993; Simion et al., 2008), and gradually gain more expertise during the first year of life. For example, whereas newborns can discriminate their mothers from strangers when the mothers' heads are uncovered (Bushneil et al., 1989), they cannot do so when both women are wearing head scarves (Pascalis et al., 1995), although this only occurs up to 5 weeks of age (Bartrip et al., 2001). Moreover, at around 7 months, infants become able to process detailed facial configurations, such as the distance between eyes and mouth (Cohen and Cashon, 2001), and to identify strangers' faces from a non-frontal view (Fagan, 1976). Discrimination of biological (e.g., a walking hen) from non-biological motion has also been observed in newborns (Simion et al., 2008), but the ability to differentiate human motion (e.g., a walking person) from nonhuman motion appears around 3 months of age (Bertenthal et al., 1987). By around 12 months of age, infants are able to discriminate possible and impossible human movements, such as fingers or elbows bending in the opposite direction (Christie and Slaughter, 2010; Morita et al., 2012). As mentioned above, although young infants already have primary discrimination abilities in relation to humans, this is not as well-developed as it is in adults. Therefore, it is likely that infant perception of humanoid robots is different from that of adults.

Investigating infant perception of androids inevitably leads to manifesting how infants discriminate human beings from nonhuman beings. Androids can be regarded as a highly controlled human stimuli for use in investigating human nature in the field of cognitive science (MacDorman and Ishiguro, 2006). Some researchers have already used androids as experimental stimuli (Saygin et al., 2011; Urgen et al., 2013); however, most targeted human adults. To our knowledge, there is only one study in which preschoolers' responses to a real human and an android were compared (Moriguchi et al., 2010), and no studies on younger infants. Therefore, the purpose of this study was to investigate infant discrimination ability in regard to human beings, using humanoid robots and the preferential looking paradigm. When two kinds of stimuli are presented simultaneously in front of infants, a remarkable difference in looking times between both stimuli indicates that infants can discriminate between each stimulus. This method was devised by Fantz in the 1950s (Fantz, 1958), and is still widely used today in the field of developmental science.

In this study, three agents-a human, an android modeled on the human, and a mechanical-looking robot made from the android-were used as the experimental stimuli. If infants can recognize relatively few differences between the human and the android, significant difference in their looking times to each agent should be observed. Taking the findings of previous studies described above into consideration, it is very likely that younger infants will not realize that the android is not a human, while infants aged over 12 months may be able to discriminate between the two; therefore, this study targeted infants aged between 6 and 14 months. Furthermore, we employed an eye tracking system to measure infant looking times because it allows for more objective measurement and more precise analysis of focused areas than manual coding does. Even if no difference is found in looking times, there may be difference in the regions infants focus on when looking at each agent. Thus, this study will provide new evidence in relation to infants' ability to discriminate human beings from non-human beings, and the pathway by which this ability develops. In addition, from the viewpoint of robotics, this experiment will evaluate the infant's perception of the human likeness of currently available androids. If the uncanny valley hypothesis applies in infancy, particular responses to the android, such as avoiding viewing the android, may be observed.

\section{Materials and Methods}

\section{Participants}

Infants ( $N=42 ; 20$ boys, 22 girls; age $=6-14$ months) were assigned to three groups based on their age: $6-8$ months (six boys, five girls, mean age $=223.73$ days, $S D=20.39$ ), 9-11 months (eight boys, nine girls, mean age $=291.63$ days, $S D=30.63$ ), and 12-14 months (six boys, eight girls, mean age $=355.39$ days, $S D=64.43$ ). A further 22 infants were excluded from analysis following cessation of the experiment due to fussiness, such as crying and inability to stay still $(n=7)$, or a lack of valid gaze data $(n=15)$. Details about the criteria for data exclusion are described in the data analysis subsection below.

This study was approved by the ethics committee of the University of Tokyo. Written informed consent was obtained from the parents of all participants before beginning the experiment.

\section{Stimuli and Apparatus}

The visual stimuli were three different black and white video clips $(800 \times 800$ pixels, $30 \mathrm{fps})$ that depicted one of three agents (a human, an android, or a mechanical robot) performing a grasping action with their right hand. Figure 1 shows example frames of each video clip. These clips were made from stimuli used in a previous study (Saygin et al., 2011).

In the human agent clip, a Japanese woman reached her right hand toward a tube of facial wash, grasped it for a moment, and then moved her hand back to the original position. Her facial 

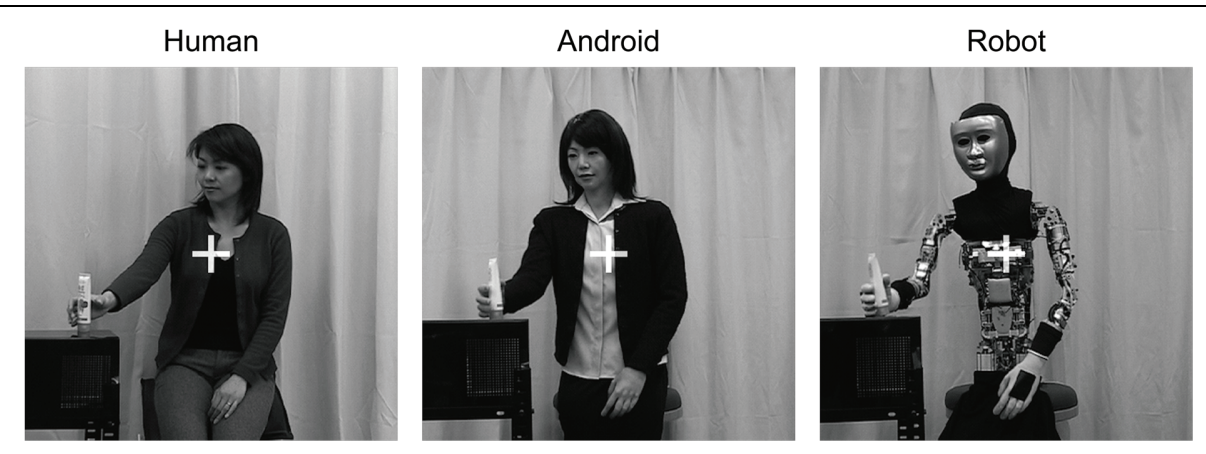

FIGURE 1 | Agents used as experimental stimuli. The android was designed to have the likeness of the human actor, and was identical in internal architecture to the robot. The original face of the robot was covered with a plastic mask to conceal its somewhat bizarre appearance, with naked eyeballs and gums.

expression did not change and her left hand remained on her left thigh. In the android and robot clips, a female android named Repliee Q2 (Osaka University and KOKORO Co. Ltd., Japan) and a mechanical humanoid robot, respectively, performed the same grasping action as the human stimulus. The Repliee Q2 was modeled on the women actor shown in the human stimulus, and its upper body is moved by air actuators. Because the mechanical robot was made by stripping away the clothing and silicone skin from the android, the robots were almost identical in terms of physical size and motion. Although the robots' motions were programed to resemble the human's action as much as possible, those were actually rather unnatural due to mechanical limitations. In more concrete terms, whereas the human moved her hand straight to the target, the robots moved their hands over the target and then down toward it. All of the video clips were $3.5 \mathrm{~s}$ in duration, the second half $(1.75 \mathrm{~s})$ of which consisted of the first half ( $1.75 \mathrm{~s}$ ) being played backwards. In addition, we used a simple animation with cheerful music that depicts a star changing in color and size as an attention getter.

Gaze data were collected at $300 \mathrm{~Hz}$ by the Tobii TX300 (Tobii AB, Sweden) contactless eye tracking system, which was placed at the center of a table. Its back and left and right sides were surrounded with curtains to ensure that the infants' concentration remained on the stimuli. The stimuli were presented on a 23 in liquid crystal display $(1920 \times 1080$ pixels $)$ integrated with the Tobii, and the actual size of each video clip on the display was a $21 \mathrm{~cm}$ square. A small video camera (CCD-MC100, Sony Corporation) was additionally attached at the center of the upper frame of the display so that we could observe participants' behavior. During gaze measurement, an experimenter who was located in an area separated by the curtain manipulated the Tobii and the stimuli.

\section{Procedure}

Infants viewed the stimuli while sitting on their parent's lap, and the distance between the infants and the display was approximately $60 \mathrm{~cm}$. The tilt angle of the Tobii was adjusted so that it only captured infants' eyes, and then a 5-point calibration was conducted. The parent was instructed not to respond to either the infant or the stimuli. In a single trial, two different video clips were presented at the same time side-by-side on the display, and were repeated three times without an interval. Thus, a single trial lasted $10.5 \mathrm{~s}$. Each pair of agents (human vs. android: HA, human vs. robot: $\mathrm{HR}$, and android vs. robot: AR) was presented four times, and the distance between two clips was $3.2 \mathrm{~cm}$. The position (left or right) of the stimuli was counterbalanced. We conducted 12 trials if the infant did not become fussy, with the presentation order of each pair randomized. Before every trial, the attention getter was played at the center of the display until the infant looked toward it. Validity of eye tracking was monitored in real time using the "Show Track Status" function of the Tobii. An experimenter determined termination of the attention getter based on this status monitor and live footage from the video camera. In addition, the experimenter asked parents to move infants back to the initial position after a trial in which the Tobii lost infants' eye gaze because they moved vigorously.

\section{Analysis}

Trials with invalid (missing) gaze data for more than $50 \%$ of the trial duration were excluded from the data analysis. Moreover, participants for whom the data of one or more agent pairs was not obtained at all, were completed excluded. There were 15 infants excluded based on this criterion, primarily due to a hardware failure of the Tobii TX300 eye tracking system. According to the developer of Tobii, when the TX300 is used with a particular firmware (ver. 1.1.0), as we did in this study, it can fail to detect infant gaze during high-frequency measurement because of a problem in its algorithm for gaze detection. This problem does not occur in measurement at lower frequencies, such as at 60 and $120 \mathrm{~Hz}$, and it has been fixed in the latest firmware (ver. 1.1.1). Regrettably, we lost a large amount of data because we were not aware of this important problem and its solution until after the experiment was complete.

We defined three static areas of interest (AOI), corresponding to the face area, a goal area, and the body area (see Figure 2). The same three AOI were applied to each agent, and statistical analysis was performed separately for each pair of agents (HA, $\mathrm{HR}$, and AR). To calculate the proportions of looking times toward each AOI of each agent, mean gaze counts were divided by the total gaze count for two agents presented simultaneously. One gaze count corresponds to $3.3 \mathrm{~ms}$ viewing at $300 \mathrm{~Hz}$ sampling. 

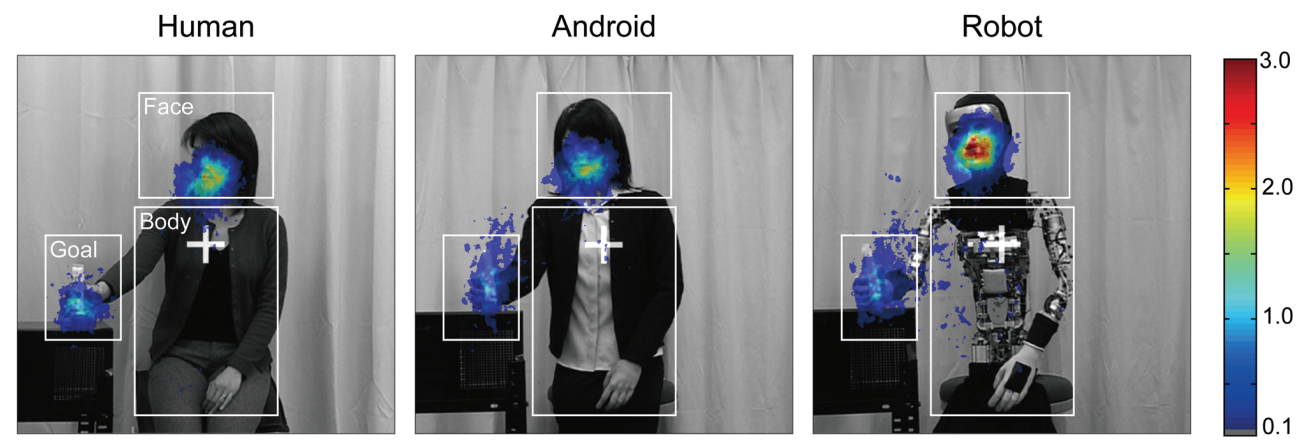

FIGURE 2 | Heat maps of mean gaze count across all trials of all participants, superimposed upon each agent after $7 \times 7$ pixel Gaussian smoothing was applied. Red represents an area that the greatest number of infants viewed. areas of interest (AOI) are depicted as white rectangles. The reason for the focused areas in the goal area of the android and the robot spreading vertically is probably due to the trajectories of the agents' hands.

A three-way mixed design analysis of variance (ANOVA; age group $\times$ agent $\times$ AOI) with the arcsine transformation was conducted for the proportions of looking times, and the HuynhFeldt correction for degrees of freedom was employed as necessary. Multiple comparison with the Bonferroni method was carried out when an interaction was found.

\section{Results}

To make it easier to understand the overall trends, heat maps of the mean gaze count across all trials of all participants for each agent are shown in Figure 2.

Figure 3 shows the mean proportions of looking time for each AOI in each age group. There were main effects of agent in the $\mathrm{HR}$ and AR conditions [HR: $F(1,39)=22.65, p<0.001$; AR: $F(1,39)=28.90, p<0.001$ ], of AOI in all three conditions [HA: $F(1.83,71.29)=45.86, p<0.001$; HR: $F(1.70,66.23)=50.64$, $p<0.001$; AR: $F(1.67,65.29)=64.46, p<0.001)$, and of age group only in the HA condition $[F(2,39)=5.83, p=0.006]$.

Moreover, an interaction between age group and AOI was found in all of the three conditions [HA: $F(3.66,71.29)=5.19$, $p=0.001$; HR: $F(3.40,66.23)=2.77, p<0.05$; AR: $F(3.35$, $65.29)=3.17, p<0.05]$. The details of significant differences between each AOI in each age group and those between each age group at each AOI are described in Tables 1 and 2, respectively. Table 1 shows that infants in all age groups principally watched the face area of each agent, and that infants aged over 9 months watched the goal area for longer than they did the body area. Further, Table 2 shows the gaze preference for the goal area in infants aged over 9 months, and shows that the 6- to 8-monthold group tended to view the body area for longer than the older groups did.

An interaction of agent and AOI was also found in the $\mathrm{HR}$ and AR conditions [HR: $F(2,78)=3.53, p<0.05$; AR: $F(2,78)=12.53, p<0.001]$. Multiple comparison revealed that the robot captured the longest looking time among all of the agents in any AOI $(p<0.05$ for the goal area in the AR condition, $p<0.01$ for the goal area in the HR condition and for the body area in the AR condition, $p<0.001$ for the rest), and that infants viewed the face area for significantly longer than they did the other AOI (all ps < 0.001).

No second-order interactions were found in any conditions. Further, no effect and interaction involved in the agent factor was
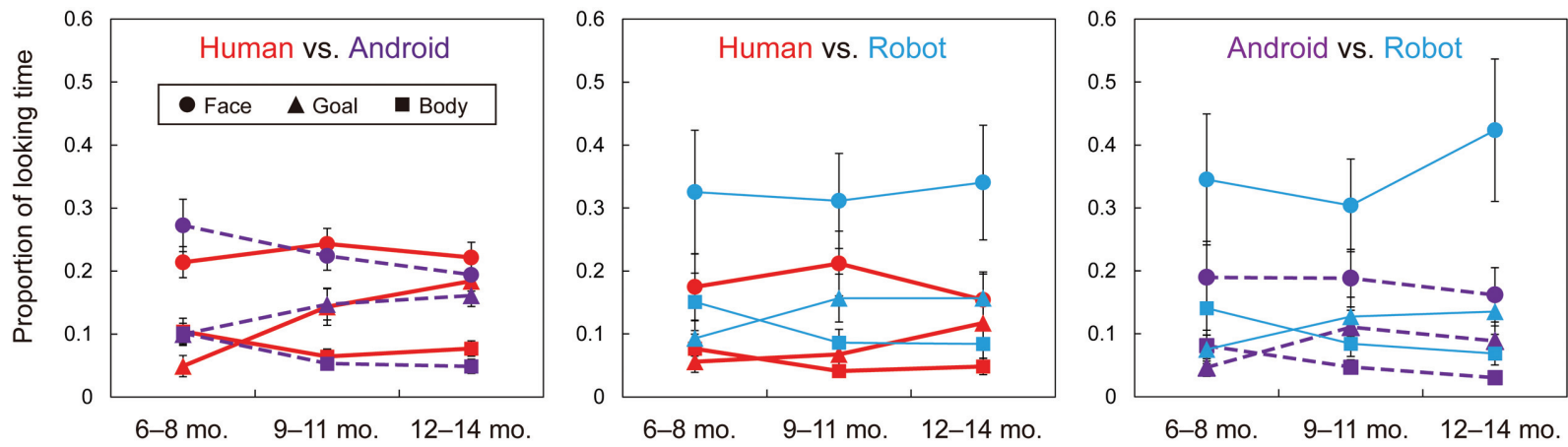

FIGURE 3 | Proportions of total looking times at each AOI of each agent across the three age groups. Red solid lines, purple dotted lines, and blue thin lines represent the human, android, and robot agents, respectively. Circle, triangle, and square markers correspond to AOI of face, goal, and body, respectively. Error bars represent standard errors. 
TABLE 1 | The results of multiple comparisons for looking times between each areas of interest (AOI; Face/Goal/Body) in each age group (6-8/9-11/12-14 months).

\begin{tabular}{|c|c|c|c|c|c|c|}
\hline Age (months) & Human vs. Android & $p<$ & Human vs. Robot & $p<$ & Android vs. Robot & $p<$ \\
\hline \multirow[t]{3}{*}{$6-8$} & Face $>$ Goal & 0.001 & Face $>$ Goal & 0.001 & Face $>$ Goal & 0.001 \\
\hline & Face $>$ Body & 0.01 & Face $>$ Body & 0.01 & Face $>$ Body & 0.001 \\
\hline & & & Body > Goal & 0.05 & & \\
\hline \multirow[t]{3}{*}{$9-11$} & Face > Goal & 0.05 & Face $>$ Goal & 0.001 & Face > Goal & 0.01 \\
\hline & Face $>$ Body & 0.001 & Face $>$ Body & 0.001 & Face $>$ Body & 0.001 \\
\hline & Goal > Body & 0.001 & Goal > Body & 0.05 & & \\
\hline \multirow[t]{3}{*}{$12-14$} & Face $>$ Body & 0.001 & Face $>$ Goal & 0.05 & Face $>$ Goal & 0.001 \\
\hline & Goal > Body & 0.001 & Face $>$ Body & 0.001 & Face $>$ Body & 0.001 \\
\hline & & & Goal > Body & 0.01 & Goal > Body & 0.05 \\
\hline
\end{tabular}

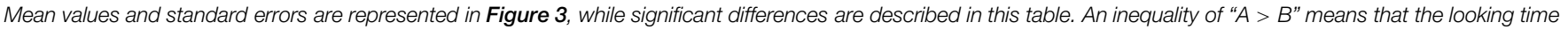
toward the $\mathrm{AOI}$ of $\mathrm{A}$ was significantly longer than that toward the $\mathrm{AOI}$ of $\mathrm{B}$.

TABLE 2 | The results of multiple comparisons for looking times between each age group (6-8/9-11/12-14 months) in each AOI (Face/Goal/Body).

\begin{tabular}{|c|c|c|c|c|c|c|}
\hline AOI & Human vs. Android & $p<$ & Human vs. Robot & $p<$ & Android vs. Robot & $p<$ \\
\hline Face & n.s. & & n.s. & & n.s. & \\
\hline \multirow[t]{2}{*}{ Goal } & $9-11>6-8$ & 0.01 & $12-14>6-8$ & 0.05 & & \\
\hline & $12-14>6-8$ & 0.001 & & & n.s. & \\
\hline \multirow[t]{2}{*}{ Body } & $6-8>9-11$ & 0.05 & $6-8>9-11$ & 0.05 & $6-8>9-11$ & 0.05 \\
\hline & & & & & $6-8>12-14$ & 0.01 \\
\hline
\end{tabular}

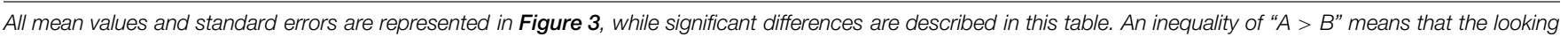
time of group A was significantly longer than that of group $B$.

detected in the HA condition; that is, there were no significant differences in either looking time or focusing area between the human and the android in any age groups.

\section{Discussion}

To examine infant discrimination ability among human and humanlike agents and to test the human likeness of a currently available android, we measured looking times of infants aged between 6 and 14 months in regard to three types of agents of similar body size and motion. The three-way ANOVA revealed that infants of all age groups spent the longest time on viewing the robot, especially its face, compared with the other agents. Further, there was no difference in looking time between the human and android agents. These results suggest that 6- to 14-month-old infants are unable to distinguish the android from the human, although they are able to distinguish the robot from the human.

Infants' gaze preference for the mechanical robot is probably derived from their novelty preference tendency. A considerable number of studies have shown that infants generally prefer unfamiliar to familiar stimuli. The fact that the preference was observed in the AR condition, where the motions of both agents were almost the same, indicates that the visual aspects of the robot, rather than the motion, captured the infants' attention. Although it is likely that the infants who participated in our experiment often saw many women besides their mother in daily life, none had seen the robot before taking part in this study; therefore, the robot must have been the most unfamiliar to them from among the three agents.
Despite the fact that the android is also a rare stimulus for the infants to have observed in reality, there was no gaze preference between the human and android agent. An absence of preference for the looking paradigm does not directly indicate that two stimuli are considered to be identical; hence, it is unclear whether the infants regarded the human and the android as the same person. However, our findings suggest, at least, that the human and the android were regarded as equally humanlike beings.

A similar insensitivity to artificial humanity in infants has been reported by a previous study (Lewkowicz and Ghazanfar, 2012), where it was exhibited that 6- to 12-month-old infants were unable to discriminate a realistic computer graphics (CG) avatar from a real human. Although the authors used the term "realistic" to describe their stimuli, the stimuli actually had a nonphotorealistic appearance that any adult could recognize as being a CG avatar at a glance. Our android had a more photorealistic appearance than theirs did; therefore, it should have been difficult for not only 6- to 12-month-old infants but also older infants to discriminate between the human and the android.

The motion of the android used in this study was unnatural due to its mechanical limitations. If infants recognize the unnaturalness of its motion, it is possible that they looked for longer at the android than at the human; however, the results showed that this was not the case. The android's grasping action is somewhat awkward but not impossible for human beings. It is likely that the discrimination ability of infants aged around 1 year for human movement is not yet sophisticated enough to detect this type of awkwardness.

In all the three conditions and for all age groups, infants spent the longest time looking at the face AOI. Infants' preference 
for faces has been reported by many previous researchers. Even newborns under 1 week of age prefer face and face-like stimuli to other stimuli (Goren et al., 1975; Macchi et al., 2004; Farroni et al., 2005), and infants gradually focus their attention on faces at between 3 and 9 months of age (Frank et al., 2009). This preference for faces has been observed regardless of the nature of the stimuli, i.e., geometric or photographic images (Farroni et al., 2005), and is, thus, considered to reflect the importance of faces in human communication (Csibra and Gergely, 2009).

Interestingly, looking times in the goal AOI were larger in the older infant groups than in the youngest group. This probably depends on the development of their prediction ability for human action. Falck-Ytter et al. (2011) compared looking behaviors of 6- and 12-month-old infants and adults while watching human goal-directed actions, and revealed that 12-month-olds and adults looked at the goal area significantly faster and for longer than 6-month-olds did. In another similar study (Kanakogi and Itakura, 2011), the authors proposed that this prediction ability for others' actions corresponds to their own motor ability, and demonstrated that infant grasping ability develops gradually after 6 months of age. Our result is highly consistent with these findings. A shorter looking at the goal AOI in the 6- to 8-monthold group may reflect their rudimentary understanding of the goal of the agents' action.

Of course, there are limitations in our study. First, it is possible that the stimuli were too small for infants to detect slight differences in appearance and motion between the human and the android. We used $21 \mathrm{~cm}$ square black and white video clips, which were presented $60 \mathrm{~cm}$ away from the infants. An agent of this size corresponds to a real agent at about $2.5 \mathrm{~m}$ distance. The presentation of a real android may produce different results. In fact, presentation at a realistic size facilitates information processing about the human body in young infants (Heron and Slaughter, 2010). Second, factors that can influence the perceived human likeness of robots are not limited to their appearance and motion. For example, a study using a mechanical humanoid robot reported that infants regarded the robot as a communicative agent only after watching interactions between

\section{References}

Alexander, G. M., Wilcox, T., and Woods, R. (2009). Sex differences in infants' visual interest in toys. Arch. Sex. Behav. 38, 427-433.

Arita, A., Hiraki, K., Kanda, T., and Ishiguro, H. (2005). Can we talk to robots? Ten-month-old infants expected interactive humanoid robots to be talked to by persons. Cognition 95, B49-B57. doi: 10.1016/j.cognition.2004.08.001

Bartrip, J., Morton, J., and Schonen, S. (2001). Responses to mother's face in 3-week to 5-month-old infants. Br. J. Dev. Psychol. 19, 219-232. doi: 10.1348/026151001166047

Bertenthal, B. I., Proffitt, D. R., and Kramer, S. J. (1987). Perception of biomechanical motions by infants: implementation of various processing constraints. J. Exp. Psychol. Hum. Percept. Perform. 13, 577-585. doi: 10.1037/0096-1523.13.4.577

Burleigh, T. J., Schoenherr, J. R., and Lacroix, G. L. (2013). Does the uncanny valley exist? An empirical test of the relationship between eeriness and the human likeness of digitally created faces. Comput. Hum. Behav. 29, 759-771. doi: $10.1016 /$ j.chb.2012.11.021

Bushneil, I., Sai, F., and Mullin, J. (1989). Neonatal recognition of the mother's face. Br. J. Dev. Psychol. 7, 3-15. doi: 10.1111/j.2044-835X.1989.tb00784.x a human and the robot (Arita et al., 2005). This finding implies that the interactive functions of robots can influence their human likeness. In addition, infants' characteristics, such as gender, and temperament, influence the perceived human likeness of robots. Because female, compared to male, infants have been reported to show an advantage in processing social stimuli, such as facial expressions (McClure, 2000), and to prefer more human-like stimuli, such as dolls and human faces (Connellan et al., 2000; Lutchmaya and Baron-Cohen, 2002; Alexander et al., 2009), their ability to discriminate between human and nonhuman beings may mature faster. Finally, gaze measurement is not the only way to investigate infant discrimination ability. Recently, infants' neural response to stimuli has been attracting attention as a new subjective index of their discrimination ability, in association with the development of non-invasive and more simplified technology for measuring brain activity (Csibra et al., 2004; Farroni et al., 2004). Although we did not find differences in infant gaze behaviors between the human and the android agents in this study, infants' neural response to the two types of agent may differ in some brain regions.

To our knowledge, this is the first report concerning infant discrimination of a recently developed android from humans and robots. Our results suggest that discrimination ability in regard to human vs. non-human beings is not as sophisticated in infants younger than 14 months as it is in adults. The uncanny valley effect elicited by the android was not found in infants; in other words, a currently available android may have already reached a humanlike quality for infants, at least with regard to appearance and motion. Androids have great potential as an alternative to human stimuli in future psychological studies.

\section{Acknowledgments}

This work was supported by JSPS KAKENHI Grant Number 25220004. We thank Dr. Yoshihiro Okazaki and Dr. Yasuhiro Kanakogi for their help in conducting the experiment.

Christie, T., and Slaughter, V. (2010). Movement contributes to infants' recognition of the human form. Cognition 114, 329-337. doi: 10.1016/j.cognition.2009.10.004

Cohen, L. B., and Cashon, C. H. (2001). Do 7-month-old infants process independent features or facial configurations? Infant Child Dev. 10, 83-92. doi: 10.1002/icd.250

Connellan, J., Baron-Cohen, S., Wheelwright, S., Batki, A., and Ahluwalia, J. (2000). Sex differences in human neonatal social perception. Infant Behav. Dev. 23, 113-118. doi: 10.1016/S0163-6383(00)0 0032-1

Csibra, G., and Gergely, G. (2009). Natural pedagogy. Trends Cogn. Sci. 13, 148-153. doi: 10.1016/j.tics.2009.01.005

Csibra, G., Henty, J., Volein, A., Elwell, C., Tucker, L., Meek, J., et al. (2004). Near infrared spectroscopy reveals neural activation during face perception in infants and adults. J. Pediatr. Neurol. 2, 85-90.

DeCasper, A. J., and Fifer, W. P. (1980). Of human bonding: newborns prefer their mothers' voices. Science 208, 1174-1176. doi: 10.1126/science.73 75928

Fagan, J. F. III. (1976). Infants' recognition of invariant features of faces. Child Dev. 47, 627-638. doi: 10.1111/j.1467-8624.1976.tb02226.x 
Falck-Ytter, T., Bakker, M., and Von Hofsten, C. (2011). Human infants orient to biological motion rather than audiovisual synchrony. Neuropsychologia 49 , 2131-2135. doi: 10.1016/j.neuropsychologia.2011.03.040

Fantz, R. L. (1958). Pattern vision in young infants. Psychol. Rec. 8, 43-47.

Farroni, T., Johnson, M. H., and Csibra, G. (2004). Mechanisms of eye gaze perception during infancy. J. Cogn. Neurosci. 16, 1320-1326. doi: 10.1162/0898929042304787

Farroni, T., Johnson, M. H., Menon, E., Zulian, L., Faraguna, D., and Csibra, G. (2005). Newborns' preference for face-relevant stimuli: effects of contrast polarity. Proc. Natl. Acad. Sci. U.S.A. 102, 17245-17250. doi: 10.1073/pnas.0502205102

Frank, M. C., Vul, E., and Johnson, S. P. (2009). Development of infants' attention to faces during the first year. Cognition 110, 160-170. doi: 10.1016/j.cognition.2008.11.010

Goren, C. C., Sarty, M., and Wu, P. Y. (1975). Visual following and pattern discrimination of face-like stimuli by newborn infants. Pediatrics 56, 544-549.

Heron, M., and Slaughter, V. (2010). Infants' responses to real humans and representations of humans. Int. J. Behav. Dev. 34, 34-45. doi: 10.1177/0165025409345047

Ishiguro, H. (2006). Android science: conscious and subconscious recognition. Conn. Sci. 18, 319-332. doi: 10.1080/095400906008 73953

Kanakogi, Y., and Itakura, S. (2011). Developmental correspondence between action prediction and motor ability in early infancy. Nat. Commun. 2, 341. doi: $10.1038 /$ ncomms 1342

Lewkowicz, D. J., and Ghazanfar, A. A. (2012). The development of the uncanny valley in infants. Dev. Psychobiol. 54, 124-132. doi: 10.1002/ dev. 20583

Lutchmaya, S., and Baron-Cohen, S. (2002). Human sex differences in social and non-social looking preferences, at 12 months of age. Infant Behav. Dev. 25, 319-325. doi: 10.1016/S0163-6383(02)00095-4

Macchi, C. V., Turati, C., and Simion, F. (2004). Can a nonspecific bias toward topheavy patterns explain newborns' face preference? Psychol. Sci. 15, 379-383. doi: 10.1111/j.0956-7976.2004.00688.x

MacDorman, K. F., and Ishiguro, H. (2006). The uncanny advantage of using androids in cognitive and social science research. Interact. Stud. 7, 297-337. doi: 10.1075/is.7.3.03mac

McClure, E. B. (2000). A meta-analytic review of sex differences in facial expression processing and their development in infants, children, and adolescents. Psychol. Bull. 126, 424-453. doi: 10.1037/0033-2909.126.3.424

Moon, C., Cooper, R. P., and Fifer, W. P. (1993). Two-day-olds prefer their native language. Infant Behav. Dev. 16, 495-500. doi: 10.1016/0163-6383(93)80007-U

Mori, M. (1970). Bukimi no tani [The uncanny valley]. Energy 7, 33-35.
Mori, M. (2012). The uncanny valley. IEEE Robot. Autom. Mag. 19, 98-100. doi: 10.1109/Mra.2012.2192811

Moriguchi, Y., Minato, T., Ishiguro, H., Shinohara, I., and Itakura, S. (2010). Cues that trigger social transmission of disinhibition in young children. J. Exp. Child Psychol. 107, 181-187. doi: 10.1016/j.jecp.2010.04.018

Morita, T., Slaughter, V., Katayama, N., Kitazaki, M., Kakigi, R., and Itakura, S. (2012). Infant and adult perceptions of possible and impossible body movements: an eye-tracking study. J. Exp. Child Psychol. 113, 401-414. doi: 10.1016/j.jecp.2012.07.003

Noma, M., Saiwaki, N., Itakura, S., and Ishiguro, H. (2006). "Composition and evaluation of the humanlike motions of an android," in Proceedings of the 2006 6th IEEE-RAS International Conference on Humanoid Robots (Genova: IEEE), 163-168. doi: 10.1109/ichr.2006.321379

Pascalis, O., De Schonen, S., Morton, J., Deruelle, C., and Fabre-Grenet, M. (1995). Mother's face recognition by neonates: a replication and an extension. Infant Behav. Dev. 18, 79-85. doi: 10.1016/0163-6383(95)90009-8

Saygin, A. P., Chaminade, T., Ishiguro, H., Driver, J., and Frith, C. (2011). The thing that should not be: predictive coding and the uncanny valley in perceiving human and humanoid robot actions. Soc. Cogn. Affect. Neurosci. 7, 413-422. doi: $10.1093 /$ scan/nsr025

Seyama, J., and Nagayama, R. S. (2007). The Uncanny Valley: effect of realism on the impression of artificial human faces. Presence 16, 337-351. doi: 10.1162 /pres.16.4.337

Simion, F., Regolin, L., and Bulf, H. (2008). A predisposition for biological motion in the newborn baby. Proc. Natl. Acad. Sci. U.S.A. 105, 809-813. doi: 10.1073/pnas.0707021105

Steckenfinger, S. A., and Ghazanfar, A. A. (2009). Monkey visual behavior falls into the uncanny valley. Proc. Natl. Acad. Sci. U.S.A. 106, 18362-18366. doi: 10.1073/pnas.0910063106

Urgen, B. A., Plank, M., Ishiguro, H., Poizner, H., and Saygin, A. P. (2013). EEG theta and $\mathrm{Mu}$ oscillations during perception of human and robot actions. Front. Neurorobot. 7:19. doi: 10.3389/fnbot.2013.00019

Conflict of Interest Statement: The authors declare that the research was conducted in the absence of any commercial or financial relationships that could be construed as a potential conflict of interest.

Copyright (c) 2015 Matsuda, Ishiguro and Hiraki. This is an open-access article distributed under the terms of the Creative Commons Attribution License (CC BY). The use, distribution or reproduction in other forums is permitted, provided the original author(s) or licensor are credited and that the original publication in this journal is cited, in accordance with accepted academic practice. No use, distribution or reproduction is permitted which does not comply with these terms. 